\title{
Liver fibrosis recognition using multi-compression elastography technique
}

\author{
Ashraf Ali Wahba, Nagat Mansour Mohammed Khalifa, Ahmed Farag Seddik, \\ Mohammed Ibrahim El-Adawy \\ Faculty of Engineering, Biomedical Engineering Department, Helwan University, Helwan, Cairo, Egypt \\ Email: ashrafwahba@h-eng.helwan.edu.eg, nagatmansour@gmail.com, ahmed sadik@h-eng.helwan.edu.eg, \\ mohamed@eladawy.com
}

Received 6 September 2013; revised 8 October 2013; accepted 19 October 2013

Copyright (C) 2013 Ashraf Ali Wahba et al. This is an open access article distributed under the Creative Commons Attribution License, which permits unrestricted use, distribution, and reproduction in any medium, provided the original work is properly cited.

\begin{abstract}
Liver fibrosis recognition is an important issue in diagnostic imaging. The accurate estimation of liver fibrosis stages is important to establish prognosis and to guide appropriate treatment decisions. Liver biopsy has been for many years the reference procedure to assess histological definition for liver diseases. But biopsy measurement is an invasive method besides it takes large time. So, fast and improved methods are needed. Using elastography technology, a correlation technique can be used to calculate the displacement of liver tissue after it has suffered a compression force. This displacement is related to tissue stiffness, and liver fibrosis can be classified into stages according to that displacement. The value of compression force affects the displacement of tissue and so affects the results of the liver fibrosis diagnosing. By using finite element method, liver fibrosis can be recognized directly within a short time. The proposed work succeeded in recognizing liver fibrosis by a percent reached in average to $86.67 \%$ on a simulation environment.
\end{abstract}

Keywords: Liver Fibrosis; Liver Cirrhosis; Liver Inflammation; Hook's Law; Correlation; Elastography and Liver Fibrosis Recognition

\section{INTRODUCTION}

This paper introduces an algorithm for recognizing liver fibrosis stage. Liver fibrosis is the imbalance of the synthesis and decomposition of the collagens and extra cellular matrix (ECM) caused by liver cell inflammation and necrosis $[1,2]$. This imbalance causes proliferation of connective tissue in the liver. In the normal liver, every gram of liver tissue contains about 5.5 to $6.5 \mathrm{mg}$ of collagen, while in cirrhotic liver it can be higher than $20 \mathrm{mg}$ [1].
When the connective tissue start to proliferate in the portal area, this process is called fibrosis, which is the liver's repairing reaction to liver cell injuries and inflammation. Cirrhosis is the end result of liver fibrosis. In the cirrhotic liver, the fibrous septa and regenerative nodule occur and the structure of the normal liver deteriorates. When fibrosis progresses to the cirrhotic stage, it can cause portal vein hypertension and many other complications. The progression of fibrosis in cirrhotic liver can push compensated liver functions to become de-compensated. When cirrhosis advances to the decompensated stage, portal vein hypertension, liver atrophy, ascites, hepatic encephalopathy, and other serious dysfunctions can lead to liver failure $[1,3]$. In order to evaluate the stage of fibrosis, liver biopsy is the main method which can be used today. According to biopsy measurements, liver inflammation can be diagnosed in grades as $A_{0}$ to $A_{3}$ where; $A_{o}$; none, $A_{1}$; mild, $A_{2}$; moderate, and $A_{3}$ is the severe inflammation. Also, the stages of fibrosis $F_{0}$ to $F_{4}$ are as follows: $F_{0}$, no fibrosis; $F_{1}$, portal fibrosis without septa; $F_{2}$, portal fibrosis with few septa; $F_{3}$, numerous septa without cirrhosis; and $F_{4}$, cirrhosis. Some previous works measure liver stiffness related to each stage of liver fibrosis and liver inflammation from liver biopsy. For liver inflammation stages in hepatitis $\mathrm{C}$ virus (HCV) mono-infected patients $A_{0}, A_{1}, A_{2}$, and $A_{3}$, the mean values of liver stiffness in Kilo Pascal $(\mathrm{KPa})$ are 4.8, 6.4, 9.4, and 12.6 respectively. Also, for liver fibrosis stages $F_{0}-F_{1}, F_{2}$, $F_{3}$, and $F_{4}$, the mean values of liver stiffness in $(\mathrm{KPa})$ are $6.6,7.4,11$, and 17.2 respectively [1]. These stiffness values for liver inflammation and liver fibrosis can be measured using elastography imaging instead of using biopsy measurements. The aim of the proposed system is to construct an algorithm which can classify between fibrosis stages according to the stiffness of each stage using medical elastography imaging. This algorithm depends on Hook's low and equation of elasticity [4-6]. By correla- 
tion between deformed and un-deformed liver images, a displacement of tissue motion can be calculated. Then, the stiffness of liver tissue can be classified according to this displacement which will vary from a stage to another in liver fibrosis. Based on that the time needed for determining, the patient liver fibrosis stage will be decreased.

\section{MATERIALS AND METHOD}

The proposed work is to determine the human liver fibrosis $\left(F_{0,1}, F_{2}, F_{3}\right.$, or $\left.F_{4}\right)$ using multi-compression force elastography technique. The proposed recognition algorithm is shown in Figure 1. This algorithm composes of the following five basic steps:

1) Initialization step.

2) Simulation of liver fibrosis images.

3) Applying of compression forces.

4) Image correlation.

5) Liver fibrosis recognition.

\subsection{Initialization Step}

The ultrasound of liver was discussed in various works [7-9]. Many previous works concentrated on studying liver fibrosis through biopsy measurements which is the main reference measurements in liver fibrosis diagnosing [10-14], and other works focused on ultrasound imaging of liver fibrosis $[15,16]$. Phantoms can be used to mimicking the soft tissue and other parts in human body, to be tested using ultrasound imaging. These phantoms used to assess the accuracy of using ultrasound imaging in tumor diagnosing [17-19]. In our proposed work we use an elastography technique which based on a multi-compression force. It is assumed that liver fibrosis stiffness

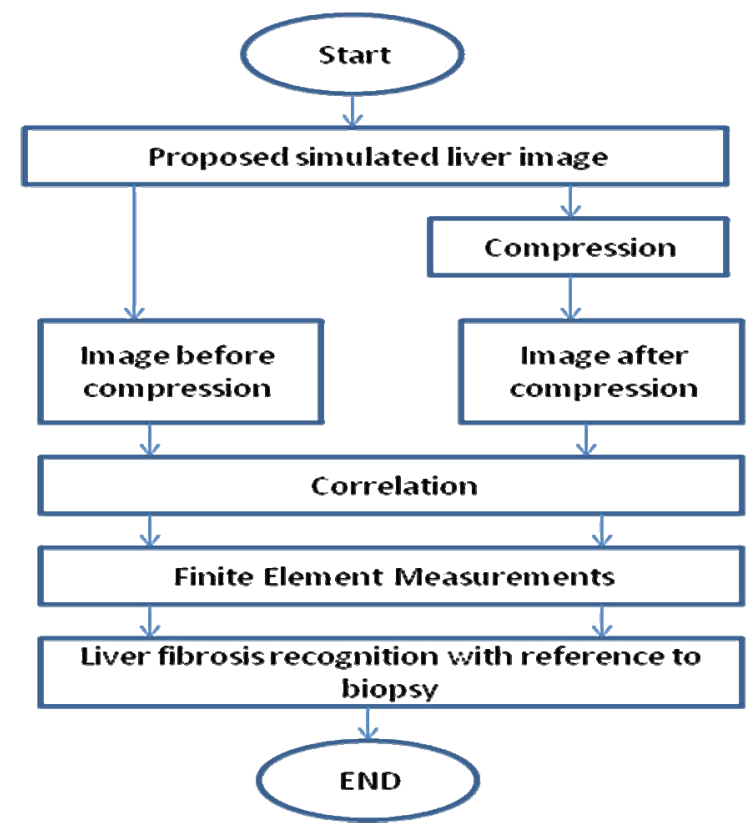

Figure 1. Block diagram of Liver fibrosis recognition. values were calculated before from biopsy measurements, and these values will be used as references when staging liver fibrosis from elastography images. Liver fibrosis stages $F_{0,1}, F_{2}, F_{3}$, and $F_{4}$ are assumed also to have stiffness values of $E_{1}, E_{2}, E_{3}$, and $E_{4}$ respectively. As stages $F_{0}$ and $F_{1}$ are near to each other, then $E_{1}$ can represent their stiffness value.

\subsection{Simulation of Liver Fibrosis Images}

Finite element method (FEM) using ABAQUS software is the source of simulated liver fibrosis images [20-22]. Finite element model of liver fibrosis is represented as shown in Figure 2. A reference material such as a silicon rubber with stiffness of $E_{r}=5(\mathrm{KPa})$ is assumed to be near that of soft tissue stiffness value to get impedance matching and good power performance. This reference material is put between the modeled soft tissue and the compression force surface to be used as a main reference layer for stiffness measurements. In ABAQUS software, the stiffness of the simulated materials are chosen, where, the stiffness of a reference material is assumed to be 5 $(\mathrm{KPa})$, soft tissue material is assumed to be $5.5(\mathrm{KPa})$, and liver fibrosis materials are assumed to be (in $\mathrm{KPa}$ ) 6.6, 7.4, 11, and 17.2 for $F_{0,1}, F_{2}, F_{3}$, and $F_{4}$ respectively [1].

\subsection{Applying of Compression Forces}

A suitable compression force is applied on the model, then, a deformation will be measured. The images before and after compression, will be taken to be correlated as described in the next section.

\subsection{Image Correlation}

Digital image processing is a main tool to describe image details and image features [23,24]. To differentiate between compressed and un-compressed images, a digital image correlation technique may be used as an important section in digital image processing [25-27].

The steps of using the two dimension (2D) digital image correlation in this proposed work are as follows:

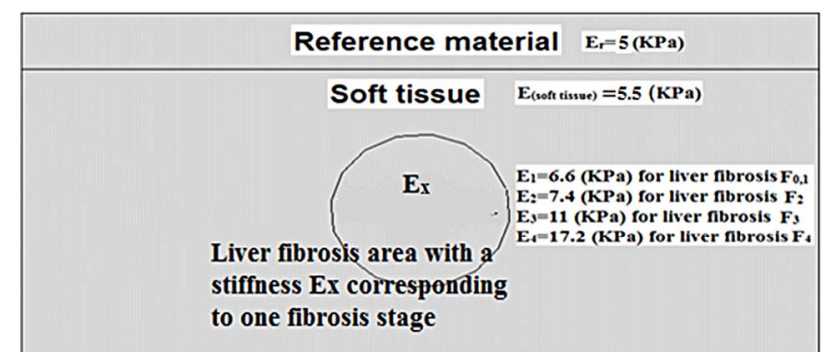

Figure 2. Representation of finite element model of liver fibrosis $\left(E_{x}\right)$, where $E_{x}$ is one of the stiffness values of $E_{1}, E_{2}, E_{3}$, or $E_{4}$. 
- Input to the correlation function $[28,29]$ the deformed (compressed) and un-deformed (un-compressed) images for correlation, and assign the first image (undeformed or un-compressed) as a reference image for correlation as shown in Figure 3. This figure contains three parts, one of them is the reference part, the second part is a soft tissue that represents the normal liver, and the third part is the liver fibroses.

- The correlation function is used to match a subset from the reference image to another in the second deformed image as shown in Figure 3 and can be written as follows in Equation (1) [28,29]

$$
R\left(x, y, x^{*}, y^{*}\right)=\sum\left|F(x, y)-G\left(x^{*}, y^{*}\right)\right|
$$

- Where $F(x, y)$ and $G\left(x^{*}, y^{*}\right)$ represent the gray levels within the subset of the un-deformed and deformed images respectively. $R$ is the magnitude of intensity value difference. Also, $(x, y)$ and $\left(x^{*}, y^{*}\right)$ are the coordinates of a point on the subset before and after deformation respectively. The symbol of the summation represents the sum of the values within the subset. The coordinate $\left(x^{*}, y^{*}\right)$ after deformation relates to the coordinate $(x, y)$ before deformation, therefore, displacement components are obtained by searching the best set of the coordinates after deformation $\left(x^{*}\right.$, $\left.y^{*}\right)$ which minimize $R\left(x, y, x^{*}, y^{*}\right)$.

- Make a grid on the reference image for the part needed to be correlated. The grid will contain a number of $(\mathrm{N})$ rasters $\left(M_{n}\right)$, where $\mathrm{n}$ varies from 0 to $\mathrm{N}-1$, and each raster $\left(M_{n}\right)$ represents number of pixels. Assuming that the motion is in one direction only $(x)$, then, the position of rasters will be in $x$ direction only and denoted by grid_ $x$.

- Run the correlation function to the previous grid. The function will give the new position of the grid rasters on the compressed image in $\mathrm{x}$ direction, which is denoted by validx.

- The displacement for each grid point $\Delta L_{x}$ in $x$ direction (the direction of the applied force) can be calculated as follows in Equation (2):

$$
\Delta L_{x}=\mid \text { grid } \_x-\text { validx } \mid
$$

\subsection{Liver Fibrosis Recognition}

Liver fibrosis stage can be determined according to the



Figure 3. Image correlation function. displacement $\Delta L_{x}$ calculated in Equation (2). Hooke's law specifies that the force affecting material is directly proportional to the displacement occurred on each part of this material as follows in Equation (3) [30].

$$
F=K \cdot X
$$

Where: $F$ is the applied force; $K$ is a constant depends on the elasticity or the stiffness of the material, and $X$ is the displacement. If the force $F$ is fixed at a constant value, then the displacement will depend only on the elasticity of the material which changes from material to another. The relation between the displacement $\Delta L_{x}$ and the stiffness $E$ is as follows in Equation (4):

$$
E=\frac{\text { Stress }}{\text { Strain }}=\frac{F / A}{\Delta L_{x} / L}=\frac{F \cdot L}{A \cdot \Delta L_{x}}
$$

Where:

$A$ is the cross section area of the material under stress, $L$ is the initial length, and $\Delta L$ is the displacement.

If $L, A$, and $F$ are assumed to be constants, then from Equation (4) we can see that the stiffness $E$ is inversely proportional to the displacement $\Delta L_{x}$ as follows in Equation (5);

$$
E \alpha \frac{1}{\Delta L_{x}}
$$

To eleminate the need for a proportionality constant we can write the stiffness of two materials as follows in Equation (6);

$$
\frac{E_{1}}{E_{2}}=\frac{\Delta L_{x 2}}{\Delta L_{x 1}}
$$

The proposed work uses different forces for compression, and with each force the displacement $\Delta L_{x n}$ of each raster $M_{n}$ will be calculated through the correlation function. This displacement of each raster will be used to indicate the liver fibrosis.

$\Delta L_{x n}$ is assumed to be the displacement of the checked raster. $\Delta L_{x r}$ is the displacement of the reference raster that has been located in the reference material which has a stiffness value of $E_{r}=5(\mathrm{KPa})$ as shown in Figure 2. Also, $E_{1}, E_{2}, E_{3}$, and $E_{4}$ are assumed to be the stiffness values to liver fibrosis stages of $F_{0,1}, F_{2}, F_{3}$, and $F_{4}$ respectively as stated above in Section 2.2. According to the proposed work, the checked rasters can be classified to refer to one of the liver fibrosis stages as follows:

Rasters that refer to fibrosis $F_{J}$ should achieve Equation (7) as follows:

$$
\frac{E_{J}}{E_{r}} \leq \frac{\Delta L_{x r}}{\Delta L_{x n}}<\frac{E_{J+1}}{E_{r}}
$$

Where $J=1,2$, or 3 .

$\left(E_{J}\right)$ is stiffness values of liver fibrosis.

$(n)$ is an index for rasters in the checked area. 
$\left(E_{r}\right)$ is the stiffness of the reference material.

$\left(\Delta L_{x r}\right)$ is the displacement of a raster in the reference material resulted from applying a compression force in $x$ direction.

$\left(\Delta L_{x n}\right)$ is the displacement of a raster in the checked area resulted from applying a compression force in $x$ direction. The above Equation (7) is suitable for fibrosis $F_{0,1}, F_{2}$, and $F_{3}$, but for rasters that refer to fibrosis $F_{4}$, it should achieve Equation (8) as follows:

$$
\frac{E_{4}}{E_{r}} \leq \frac{\Delta L_{x r}}{\Delta L_{x n}}
$$

The correct rasters and then the correct liver fibrosis stage will be recognized, and the success of our algorithm is represented by a ratio called correct recognition ratio (CRR), which specifies the number of liver fibrosis image rasters that can be recognized correctly. Multiple compression forces can be assumed and the liver fibrosis will be recognized for each compression force magnitude, and an average correct recognition ratio can be calculated.

\section{RESULTS}

To calculate the correctness of classification between different fibrosis stages we will follow these steps:

1) In the FEM domain we will consider set up of three areas, known as a reference material, soft tissue, and a known fibrosis area as shown in Figure 2.

2) Consider 100 rasters distributed in each of the three areas where the position of each raster in these areas is known.

3) Apply a compression force in the direction from the reference material to the soft tissue to the fibrosis area. As results of the force, each raster will move certain displacement in the direction of the applied force depending on the material stiffness that contains this raster.

4) Use the correlation technique to recognize each raster's new position after the force is applied.

5) From this new position of each raster, the displacement of this raster will be calculated.

6) Applying Equations (7) and (8) on the displacement calculated in step 5 which depending on the magnitude of the applied force, the type of the material can be identified which may be one of the following materials;

a) Reference material.

b) Soft tissue.

c) Fibrosis $F_{1}$.

d) Fibrosis $F_{2}$.

e) Fibrosis $F_{3}$.

f) Fibrosis $F_{4}$.

7) According to the classification of each raster material we fill in Table 1, where from this table we can calculate the correct recognition ratio CRR for those 100 checked rasters affected by that specific force.

8) Changing the force and go to step 3) and repeat for 10 different values of the applied force, and in each case calculate CRR.

9) Calculate the overall CRR on the 10 different forces.

10) Table 2 shows the average of the 10 tables where each one represents certain force.

Table 1. CRR for liver fibrosis assuming 100 rasters in each area.

\begin{tabular}{|c|c|c|c|c|c|c|c|c|}
\hline \multirow{2}{*}{\multicolumn{2}{|c|}{$\begin{array}{c}\text { Recognized area } \\
\text { Actual area } \\
\end{array}$}} & \multirow{2}{*}{ Reference } & \multirow{2}{*}{ Soft tissue } & \multicolumn{4}{|c|}{ Fibrosis } & \multirow{2}{*}{ CRR (\%) } \\
\hline & & & & $F_{1}$ & $F_{2}$ & $F_{3}$ & $F_{4}$ & \\
\hline $\mathrm{Re}$ & & 90 & 5 & 4 & 1 & - & - & 90 \\
\hline Sof & & 3 & 95 & 2 & - & - & - & 95 \\
\hline \multirow{4}{*}{ Fibrosis } & $F_{1}$ & - & 3 & 80 & 10 & 7 & - & 80 \\
\hline & $F_{2}$ & - & - & 4 & 90 & 6 & - & 90 \\
\hline & $F_{3}$ & - & - & - & 12 & 85 & 3 & 85 \\
\hline & $F_{4}$ & - & - & - & 7 & 8 & 85 & 85 \\
\hline \multicolumn{8}{|c|}{ Average correct recognition } & 85 \\
\hline
\end{tabular}

Table 2. Overall CRR for each liver fibrosis as a result of applying 10 different values of the compression force.

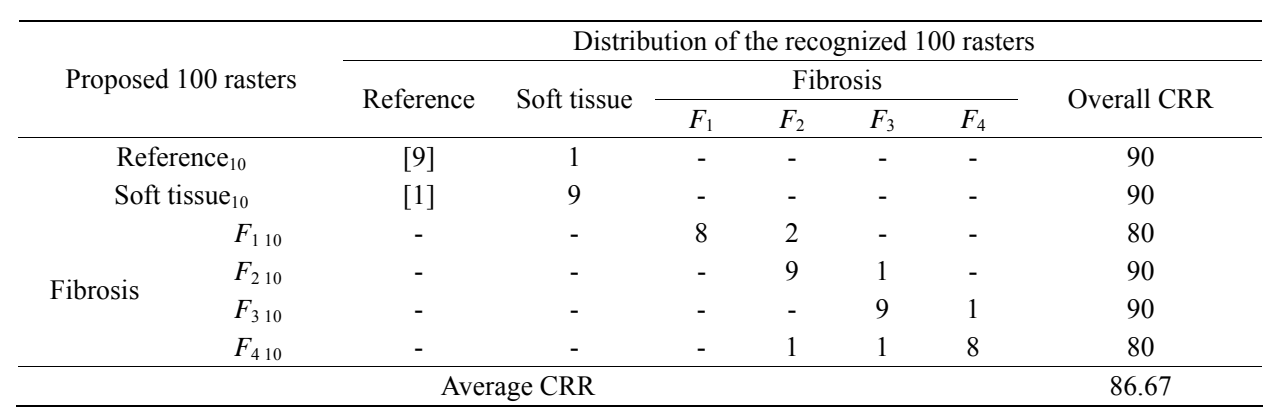




\section{CONCLUSION}

The liver fibrosis can be recognized in the elastography imaging by using liver fibrosis biopsy measurements as a reference. The biopsy measurements give the average stiffness values of liver fibrosis. The equation of elasticity specifies that tissues with high stiffness values will move a short distance when it is exposed to a certain compression force, and vice versa. The proposed recognition algorithm of liver fibrosis stages takes a short time to recognize the liver fibrosis stage. This algorithm can recognize the liver fibrosis stages $F_{0,1}, F_{2}, F_{3}, F_{4}$, by an overall CRR of about $86.67 \%$. This method of course will be faster than biopsy measurements, besides it is considered as a non-intervening operation. This proposed work can be improved by using real elastography images for liver fibrosis, then, the correct recognition ratio CRR is expected to be increased. It is worth to mention that the tolerance in each of the reference values of liver fibrosis stiffness is being ignored in this proposed work to avoid complicated calculations, and that resulted in decreasing of CRR. This shortcoming can be eliminated in the future work.

\section{REFERENCES}

[1] Eugenia, V., Pablo, B., et al. (2009) Over estimation of liver fibrosis staging using transient elastography in patients with chronic hepatitis $\mathrm{C}$ and significant liver inflammation. International Medical Press, 1359-6535.

[2] Silver, D., Karnik, G. and Osinusi, A. (2013) Effect of HIV on liver fibrosis among $\mathrm{HCV}$-infected African Americans. http://cid.oxfordjournals.org/

[3] Mercedes, de T. and Thierry, P. (2003) Risk factors for liver fibrosis progression in patients with chronic hepatitis C. Annals of Hepatology, 2, 5-11.

[4] Eric, J.C., Jan, N., et al. (1996) Young's modulus measurements of soft tissues with application to elasticity imaging. IEEE Transducers on Ultrasonics, Ferroelectrics, and Frequency Control, 43.

[5] Wen, C., Jeng, Y.-M., et al. (2001) Young's modulus of human liver and correlation with pathological findings. Department of Electrical Engineering, National Taiwan University, IEEE, Ultrasonics Symposium, 1233.

[6] Mauro, M.S., Juan, F.C., et al, (2007) Comparing optimization algorithms for young's modulus reconstruction in ultrasound elastography. Mechanical Department Katholieke Universiteit, Leuven, IEEE Ultrasonics Symposium.

[7] Christoph, F.D., Carla, S. and Maciej, J. (2011) Ultrasound of the liver. European Course Book by University of Bologna, Department of Diagnostic Imaging, 2nd Medical Faculty of Warsaw Medical University.

[8] Ben, A., Claire, R.L. and Sameer, K. (2009) Imaging of liver cancer. World Journal of Gastroenterology, The WJG Press and Baishideng, 1007-9327.
[9] Harald, L. and Elisabetta, B. (2011) Manual of diagnostic ultrasound. 2nd Edition, World Health Organization.

[10] Yves, B., Marie, B., Vincent, Di M., Frederic, C., Felipe, A., Anne, C., Michel, V., Francois, B., Pierre, O., Christine, K. and Thierry, P. (1999) Liver fibrosis progression in human immunodeficiency virus and hepatitis $\mathrm{C}$ virus coinfected patients. The Multivirc Group Hepatology, 30.

[11] Mircea, G. (2006) Noninvasive biochemical markers of liver fibrosis. Journal of Gastrointestin Liver, 15, 149159.

[12] Ayman, K. and Samir, S. (2011) Data mining visualization to support biochemical markers for liver fibrosis in patients with chronic hepatitis C virus. International Journal of Artificial Intelligence and Expert Systems (IJAE), 2.

[13] Abdelfattah, M.A., Sanaa, O.A. and Ahmed, A.A. (2013) Diagnostic value of fibronectin discriminant score for predicting liver fibrosis stages in chronic hepatitis $\mathrm{C}$ virus patients. Annals of Hepatology, 12, 44-53.

[14] Xavier, F., Sergi, A. and Josep, M.L. (2002) Identification of chronic hepatitis c patients without hepatic fibrosis by a simple predictive model. American Association for the Study of Liver Diseases. Hepatology, 36.

[15] Yu, I., Hiroshi, E., Tadashi, Y. and Hiroyuki, H. (2009) A method of liver fibrosis estimation based on combination of Rayleigh distributions. Proceedings of Symposium on Ultrasonic Electronics, 30, pp. 343-344.

[16] Monica, L., Sergiu, N. and Cristian, V. (2007) Estimating the fibrosis stage in the human liver tissue using image processing methods on ultrasonographic images. Proceedings of the 3rd International Conference-EMMIT.

[17] Doris, N. and Jayant, A.T. and Copyright, V.C. (2011) Noninvasive assessment of liver fibrosis. The American Association for the Study of Liver Diseases, Hepatology.

[18] Cannon, S., Browne, L. and Fagan, J. (2010) Assessment of the accuracy of an ultrasound elastography liver scanning system using a PVA-cryogel phantom with optimal acoustic and mechanical properties. Physics in Medicine and Biology, 55, 5965-5983. http://dx.doi.org/10.1088/0031-9155/55/19/022

[19] Sandrin, L., Oudry, J. and Bastard, C. (2011) Non-invasive assessment of liver fibrosis by vibration-controlled transient elastography $\left(\right.$ Fibroscan $\left.{ }^{\circledR}\right)$. www.intechopen.com

[20] (2012) ABAQUS installation and licensing guide. http://www.3ds.com/products/simulia/overview/

[21] Division of Engineering Brown University (2012) ABAQUS tutorial. EN175: Advanced mechanics of solids. http://www.brown.edu/Departments/Engineering/Courses /En175/

[22] Product of Dassault Systèmes Simulia Corp. (2011) Abaqus 6.11 Analysis User's Manual Volume V, Prescribed Conditions, Constraints \& Interactions. Dassault Systèmes.

[23] Amalka, P. (2009) Digital image analysis of cells. Applications in 2D, 3D and time. Box 337, SE-75105 Uppsala, Sweden. Faculty of Science and Technology, Centre for Image Analysis, Uppsala University, Uppsala, Pinidiyaa- 
rachchi.

[24] Thomas, M.D. (2011) Fundamentals of biomedical image processing. Biomedical Engineering, Springer-Verlag, Berlin/Heidelberg.

http://dx.doi.org/10.1007/978-3-642-15816-2__1

[25] Zhang, D.S. and Dwayne, D.A. (2004) Applications of digital image correlation to biological tissues. Journal of Biomedical Optics, 9, 691-699. http://dx.doi.org/10.1117/1.1753270

[26] Su, C. and Anand, L. (2003) A new digital image correlation algorithm for whole field displacement measurement. Department of Mechanical Engineering Massachusetts Institute of Technology Cambridge.
[27] Tong, W. (2005) An evaluation of digital image correlation criteria for strain mapping applications. Yale University, Department of Mechanical Engineering, New Haven, Blackwell Publishing Ltd., Strain, 167-175.

[28] Christoph, E., Robert, T. and Daniel, G. (2012) Digital image correlation and tracking with matlab. http://www.mathworks.com/matlabcentral/fileexchange/1 2413-digital-image-correlation-and-tracking

[29] Satoru, Y. and Go, M. (2007) Digital image correlation. Experimental mechanics, encyclopedia of life support systems (EOLSS).

[30] Alberto, C. and Sergio, C.M. (2010) The law of elasticity. University of Padua, Psicolo'gica, 647-657. 